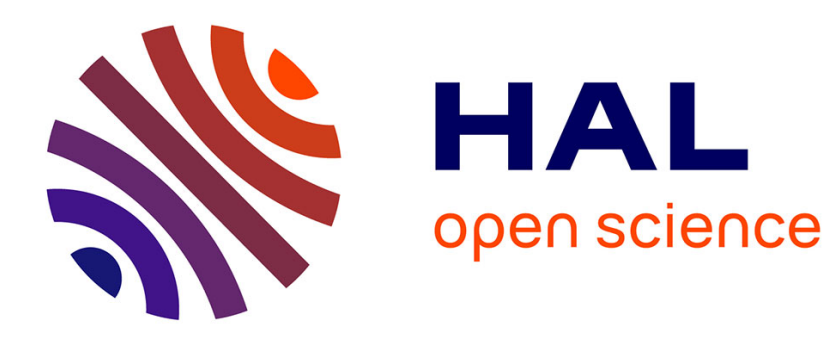

\title{
Multimodal galloping of dense spectra structures
}

\author{
Angelo Luongo, Fabio Di Fabio
}

\section{To cite this version:}

Angelo Luongo, Fabio Di Fabio. Multimodal galloping of dense spectra structures. Journal of Wind Engineering and Industrial Aerodynamics, 1993, 48 (2-3), pp.163-174. hal-00802615

\section{HAL Id: hal-00802615 https://hal.science/hal-00802615}

Submitted on 20 Mar 2013

HAL is a multi-disciplinary open access archive for the deposit and dissemination of scientific research documents, whether they are published or not. The documents may come from teaching and research institutions in France or abroad, or from public or private research centers.
L'archive ouverte pluridisciplinaire HAL, est destinée au dépôt et à la diffusion de documents scientifiques de niveau recherche, publiés ou non, émanant des établissements d'enseignement et de recherche français ou étrangers, des laboratoires publics ou privés. 


\title{
Multimodal galloping of dense spectra structures
}

\author{
A. Luongo and F. Di Fabio
}

Dipartimento di Ingegneria delle Strutture, delle Acque e del Terreno, Università degli Studi di L'Aquila, Monteluco di Roio, AQ, Italy

\section{Summary}

Nonlinear interaction phenomena among galloping modes of slender structures having several frequencies contained in one or more bands are analyzed. Due to nonlinear modal coupling associated with aerodynamic forces, all the modes of a band are in internal resonance. By referring to a nearly-periodic system consisting of weakly coupled beams and using the multiple scale perturbation method, a system of nonlinear differential equations in the amplitudes and phases of the interactive modes is obtained. Numerical results relative to a two-beam system are presented. In particular, the conditions under which steady-state solutions can occur are determined and their stability is investigated, while the occurrence of periodic motions involving exchanges of energy among the interactive modes is also remarked upon. Attention is given to the influence of small imperfections causing asymmetry of the structure.

\section{Introduction}

Galloping phenomena in prismatic structures both in the linear and nonlinear fields have been widely studied [4-7]. In the classical approach the structure is considered to oscillate with an assumed modal shape (monomodal galloping), the aerodynamic forces being expressed by the quasi-static theory. Linear analysis allows determination only of the critical velocity of galloping, i.e. the value at which selfexcited oscillations are triggered. The nonlinear theory also permits investigation of the postcritical behavior, describing the transient and steady-state regimes through the classical asymptotic methods of nonlinear mechanics.

Mathematical and experimental models have also been employed to analyze nonlinear interaction between two modes (bimodal galloping), one flexural the other torsional [1] or both flexural [8]. These studies have shown that if the two modes are in a state of internal resonance, strong coupling 
occurs in the postcritical range. Internal resonance is encountered when the natural frequencies $\omega_{1}$ and $\omega_{2}$ of the linearized system are in suitable integer ratios. If the aerodynamics nonlinearities are assumed to be of the cubic type, internal resonance takes place when $\omega_{1} / \omega_{2}=1,3,1 / 3$. The resonance $\omega_{1}=\omega_{2}$ has been found to entail the strongest coupling.

In this work the nonlinear interaction among two or more galloping modes (multimodal galloping) are studied. To this end elastic structures with dense spectra are considered, i.e. systems having a high number of frequencies contained in one or more bands. Thus, all the modes associated with the frequencies of the band are near to the internal resonance condition of the $1: 1$ type and therefore all are involved in the aeroelastic phenomenon.

The analysis is developed by reference to a chain of $n$ cantilevers weakly coupled by a soft elastic spring. By applying the quasi-static theory and expanding the aerodynamics nonlinearities up to cubic terms in the velocities, $n$ modal second-order equations are obtained. By using the multiple scale perturbation method $2 n$ differential first-order equations ensue; these allow evaluation of amplitude and phase modulating functions. Numerical results are so far limited to a two-beam system, similar to that of Pheinsusom et al.; nevertheless, some new more detailed findings have emerged here. Two different systems are considered, one symmetrical the other asymmetrical; in both cases a study is made of steady-state solutions, their stability and transient regime.

\section{Equations of motion}

Let us consider the system illustrated in Fig. 1, made of $n$ clamped-free beams coupled by elastic springs. Let the mechanical, geometrical and inertial properties of the beams differ by small quantities (for example due to imperfections) and let the stiffness $k_{\mathrm{c}}$ of the spring be small in comparison with the nominal bending stiffness of the beams. The structure is therefore a nearly-periodic weakly-coupled system. It is well-known [2] that such a system has a band spectrum; the smaller $k_{\mathrm{c}}$ the narrower the bandwidth. In a given band all the

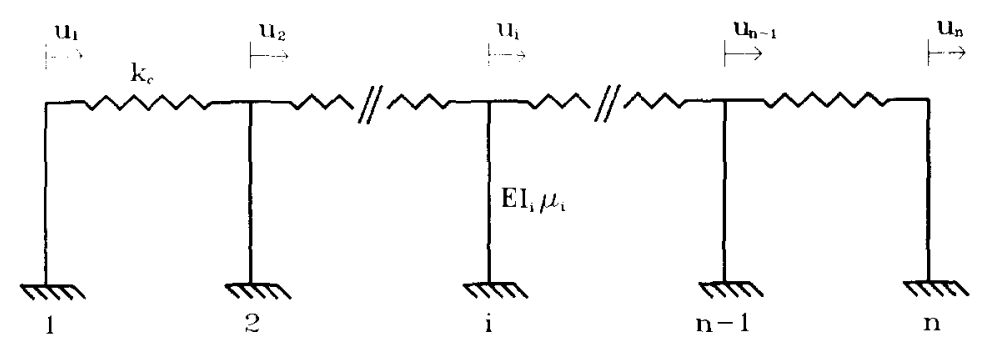

(a)

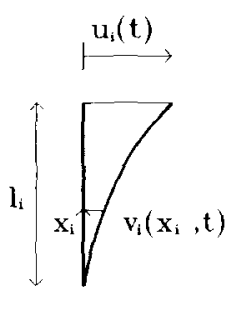

(b)

Fig. 1. (a) Model; (b) Displacements. 
beams oscillate with practically the same shape $\psi(x)$ of the isolated cantilever; thus the continuous system can be reduced to an $n$ DOF system. This is accomplished by assuming $v_{i}(x, t)=\psi(x) u_{i}(t)$, where $v_{i}$ is the transversal displacement at the nondimensional abscissa $x$ of the $i$-th beam and $u_{i}$ a function of time $t$ only, for example the displacement of the tip $x=1$, if $\psi(1)=1$.

Let the wind flow normally to the plane structure. Assuming that any interference phenomena can be neglected and applying the quasi-static theory, the aerodynamic forces in the plane can be expressed as

$f_{i}^{\mathrm{a}}(x, t)=\frac{1}{2} \rho V^{2} D_{i}\left[\frac{\mathscr{A}_{1 i}}{V} \dot{v}_{i}(x, t)+\frac{\mathscr{A}_{3 i}}{V^{3}} \dot{v}_{i}^{3}(x, t)\right]$,

where $\rho$ is the air density, $V$ the wind velocity, $D_{i}$ a dimension of the $i$-th beam cross-section and $\mathscr{A}_{k i}$ aerodynamic coefficients. Quadratic coefficients $\mathscr{A}_{2 i}$ have been assumed to be zero.

By applying the generalized Hamilton principle, the following $n$ secondorder ordinary differential equations in the $u_{\mathrm{i}}$ unknowns are obtained

$$
M \ddot{\boldsymbol{u}}+C_{\mathrm{v}} \dot{\boldsymbol{u}}+K \boldsymbol{u}=C_{\mathrm{a}} \dot{\boldsymbol{u}}+\boldsymbol{f}\left(\dot{\boldsymbol{u}}^{3}\right)
$$

In Eq. (2) $M$ is the diagonal mass matrix, $K$ the stiffness matrix, $C_{\mathrm{v}}$ and $C_{\mathrm{a}}$ the diagonal damping and aerodynamic matrices respectively, and $f$ is the vector of the nonlinear forces. Note that geometrical and/or mechanical nonlinearities of the structure have been neglected in comparison with the aerodynamic nonlinearities.

By introducing the linear transformation $u=\Phi q$, where $\Phi=\left[u_{i j}\right]$ is the modal matrix of the structure (in the absence of aerodynamic effects) and $q$ is the vector of the modal coordinates, the following equations are obtained

$\ddot{q}_{i}+\omega_{i}^{2} q_{i}=c_{i} \dot{q}_{i}+\sum_{j=1}^{n} \sum_{k=1}^{n} \sum_{l=1}^{n} c_{i j k l} \dot{q}_{j} \dot{q}_{k} \dot{q}_{l} \quad(i=1,2, \cdots, n)$,

$\omega_{i}$ being the $i$-th natural frequency. Coefficients $c_{i}$ and $c_{i j k l}$ depend on the wind velocity and are given in the Appendix. It should be noted that out-of-diagonal linear terms of the type $c_{i j} \dot{q}_{j}$ have been neglected, because they vanish when imperfections disappear. This approximation is consistent with the perturbative solution developed below.

\section{Perturbation analysis}

Nonlinear Eqs. (3) are asymptotically solved for small amplitudes by using the multiple scale perturbation method [3]. The equations are first rewritten by multiplying the r.h.m. by a perturbation parameter $\varepsilon \ll 1$. This entails assuming amplitudes of order $\varepsilon^{1 / 2}$ and coefficients $c_{i}$ of order $\varepsilon$, i.e. wind velocities near the critical values (for which $c_{i}=0$ ). The unknowns are then expanded in series of $\varepsilon$

$q_{i}(t)=q_{i 0}\left(T_{0}, T_{1}, \ldots\right)+\varepsilon q_{i 1}\left(T_{0}, T_{1}, \cdots\right)+\cdots$ 
where $T_{0}=t, T_{1}=\varepsilon t, \cdots$, are independent time scales. By using the chain rule $\frac{\mathrm{d}}{\mathrm{d} t}=D_{0}+\varepsilon D_{1}+\cdots, \quad \frac{\mathrm{d}^{2}}{\mathrm{~d} t^{2}}=D_{00}^{2}+2 \varepsilon D_{01}^{2}+\cdots$,

where $D_{k}=\partial / \partial T_{k}, D_{j k}^{2}=\partial^{2} / \partial T_{j} \partial T_{k}$, the following perturbation equations are obtained

$D_{00}^{2} q_{i 0}+\omega_{i}^{2} q_{i 0}=0$

$D_{00}^{2} q_{i 1}+\omega_{i}^{2} q_{i 1}=-2 D_{01}^{2} q_{i 0}+c_{i} D_{0} q_{i 0}+\sum_{j, k, l}\left(D_{0} q_{j 0}\right)\left(D_{0} q_{k 0}\right)\left(D_{0} q_{t 0}\right)$

Eq. (6a) admits the generating solution

$q_{i 0}=A_{i}\left(T_{1}, \cdots\right) \exp \left(\mathrm{i} \omega_{i} T_{0}\right)+$ c.c.,

where $A_{i}$ is the complex amplitude and c.c. stands for complex conjugate terms. By substituting Eq. (7) in the r.h.m. of Eq. (6b), harmonic terms of frequencies $\omega_{i}, \omega_{j} \pm \omega_{k} \pm \omega_{l}$ are obtained. Due to the fact that the $n$ frequencies are nearly coincident, i.e.

$\omega_{i}=\omega_{1}+\varepsilon \sigma_{i} \quad(i=2, \cdots n)$

these terms are resonant. There exists therefore combination resonance between all the modes of the system.

To remove secular terms the following condition must be satisfied

$$
\begin{aligned}
2 \omega_{i} D_{1} A_{i}= & c_{i} \omega_{i} A_{i}+\sum_{j . k . l} c_{i j k l} \omega_{j} \omega_{k} \omega_{l}\left[A_{j} A_{k} \bar{A}_{l} \exp \left(\mathrm{i} \sigma_{j k l i} T_{1}\right)\right. \\
& \left.+A_{j} \bar{A}_{k} A_{l} \exp \left(\sigma_{j l k i} T_{1}\right)+\bar{A}_{j} A_{k} A_{l} \exp \left(\mathrm{i} \sigma_{k l j i} T_{1}\right)\right]
\end{aligned}
$$

in which $\sigma_{j k l i}=\sigma_{j}+\sigma_{k}-\sigma_{l}-\sigma_{i}$ and the overbar denotes the complex conjugate. By expressing the amplitudes in the polar form

$$
A_{i}\left(T_{1}\right)=\frac{1}{2} a_{i}\left(T_{1}\right) \exp \left[\mathrm{i} \theta_{i}\left(T_{1}\right)\right]
$$

and zeroing the real and imaginary parts of Eqs. (9), $2 n$ differential equations of the first order in the unknowns $a_{i}\left(T_{1}\right), \theta_{i}\left(T_{1}\right)$ are obtained. To make the system autonomous the $n-1$ functions

$\ddot{i i}_{i}=\theta_{i}+\sigma_{i} T_{1}-\theta_{1} \quad(i=2,3, \cdots n)$

are introduced, each representing the phase difference at time $t$ between $q_{i} \approx q_{i 0}$ and $q_{1} \approx q_{10}$. In this way the following equations are drawn

$$
\begin{aligned}
a_{i}^{\prime}= & \frac{1}{2} c_{i} a_{i}+\frac{1}{8 \omega_{i}} \sum_{j . k . l} \omega_{j} \omega_{k} \omega_{l} c_{i j k l} a_{j} a_{k} a_{l}\left[\cos \left(\gamma_{j}+\eta_{l}-\gamma_{k}-\gamma_{i}\right)\right. \\
& \left.+\cos \left(\gamma_{j}+\gamma_{k}-\gamma_{l}-\gamma_{i}\right)+\cos \left(\gamma_{k}+\gamma_{1}-\gamma_{j}-\gamma_{i}\right)\right] \quad(i=1,2, \cdots n)
\end{aligned}
$$




$$
\begin{aligned}
a_{1} a_{i} \gamma_{i}^{\prime}= & a_{1} a_{i} \sigma_{i}+\frac{a_{1}}{8 \omega_{i}} \sum_{j, k, l} \omega_{j} \omega_{k} \omega_{l} c_{i j k l} a_{j} a_{k} a_{l}\left[\sin \left(\gamma_{j}+\gamma_{l}-\gamma_{k}-\gamma_{i}\right)\right. \\
& \left.+\sin \left(\gamma_{k}+\gamma_{j}-\gamma_{l}-\gamma_{i}\right)+\sin \left(\gamma_{l}+\gamma_{k}-\gamma_{j}-\gamma_{i}\right)\right] \\
& -\frac{a_{i}}{8 \omega_{1}} \sum_{j, k, l} c_{1 j k l} a_{j} a_{k} a_{l}\left[\sin \left(\gamma_{j}-\gamma_{k}+\gamma_{l}\right)\right. \\
& \left.+\sin \left(\gamma_{l}-\gamma_{j}+\gamma_{k}\right)+\sin \left(\gamma_{k}+\gamma_{l}-\gamma_{j}\right)\right] \quad(i=2,3, \cdots n)
\end{aligned}
$$

that can be numerically integrated to find the amplitude and phase modulation of the solution (7).

Eqs. (12) represent a nonlinear dynamic system of state variable $\left\{a_{i}, \gamma_{i}\right\}$ asymptotically equivalent to the original system of state variable $\left\{q_{i}, \dot{q}_{i}\right\}$. Although the dimensions of the two problems are practically the same, integrating in state space $\left\{a_{i}, \gamma_{i}\right\}$ is much simpler than integrating in modal coordinates space. In fact the $a_{i}$ and $\gamma_{i}$ are much slower functions of time than $q_{i}$ 's. In addition Eqs. (12) allow determination of the steady-state solutions (unmodulated solutions) simply as equilibrium points $a_{i}^{\prime}=\gamma_{i}^{\prime}=0$, corresponding to limit cycles in the modal space. This entails the solution of a nonlinear algebraic problem. Finally, by linearizing the motion around an equilibrium point, its stability or instability can be predicted by analyzing the eigenvalues of the variational matrix.

\section{Numerical results: a two-beam system}

Preliminary numerical results are obtained for a two-beam system. In this case Eqs. (12) read

$$
\begin{gathered}
a_{1}^{\prime}=c_{1} a_{1}+3 c_{2} a_{1}^{3}+3 c_{3} a_{1}^{2} a_{2} \cos \gamma+c_{4} a_{1} a_{2}^{2}(2+\cos 2 \gamma)+3 c_{5} a_{2}^{3} \cos \gamma \\
a_{2}^{\prime}=d_{1} a_{2}+3 d_{2} a_{1}^{3} \cos \gamma+d_{3} a_{1}^{2} a_{2}(2+\cos 2 \gamma)+3 d_{4} a_{1} a_{2}^{2} \cos \gamma+3 d_{5} a_{2}^{3} \\
a_{1} a_{2} \gamma^{\prime}=a_{1} a_{2} \sigma-a_{1}\left(3 d_{2} a_{1}^{3} \sin \gamma+d_{3} a_{1}^{2} a_{2} \sin 2 \gamma+d_{4} a_{1} a_{2}^{2} \sin \gamma\right) \\
\quad-a_{2}\left(c_{3} a_{1}^{2} a_{2} \sin \gamma+c_{4} a_{1} a_{2}^{2} \sin 2 \gamma+3 c_{5} a_{2}^{3} \sin \gamma\right),
\end{gathered}
$$

where $\gamma=\gamma_{2}, \sigma=\sigma_{2}$ and coefficients $c_{i}$ and $d_{i}$ are defined in the Appendix. If the system is symmetrical (identical beams) some coefficients vanish and the equations simplify as follows

$$
\begin{aligned}
& a_{1}^{\prime}=c_{1} a_{1}+3 c_{2} a_{1}^{3}+c_{4} a_{1} a_{2}^{2}(2+\cos 2 \gamma) \\
& a_{2}^{\prime}=d_{1} a_{2}+d_{3} a_{1}^{2} a_{2}(2+\cos 2 \gamma)+3 d_{5} a_{2}^{3} \\
& a_{1} a_{2} \gamma^{\prime}=a_{1} a_{2}\left[\sigma-\left(d_{3} a_{1}^{2}+c_{4} a_{2}^{2}\right) \sin 2 \gamma\right]
\end{aligned}
$$

(see also Ref. [8], where similar equations were obtained). If Eqs. (13) or (14) are linearized, equations in $a_{1}$ and $a_{2}$ uncouple and the classical monomodal solution is recovered, according to which amplitudes grow (decay) exponentially if $c_{1}$ and $d_{1}$ are positive (negative). The wind velocities $V_{\mathrm{cr} 1}$ and $V_{\mathrm{cr} 2}$ for 
which $c_{1}=0$ and $d_{1}=0$, respectively, are the critical galloping velocities associated with the two modes of the structure (Den Hartog criterion, Ref. [5]). In the postcritical range $V>V_{\mathrm{cr} 2}>V_{\mathrm{cr} 1}$ both modes are excited: when the amplitudes become moderately large nonlinearities govern the phenomenon and involve coupling between the modes.

In the following the postcritical behavior of a symmetric and an asymmetric structure is investigated.

\subsection{Symmetrical system}

The structure analyzed is made of two square-box beams with edge $D$ and thickness $s$, having the following characteristics: $D=0.2 \mathrm{~m}, s=0.02 \mathrm{~m}, l=7 \mathrm{~m}$, $I=1.42 \times 10^{-5} \mathrm{~m}^{4}, E=2 \times 10^{11} \mathrm{~N} / \mathrm{m}^{2}, \mu=16.5 \mathrm{Kg} / \mathrm{m}$; the aerodynamic coefficients are $\mathscr{A}_{1}=2.69$ and $\mathscr{A}_{3}=-168[5]$; the stiffness of the spring is $k_{\mathrm{c}}=550 \mathrm{~N} / \mathrm{m}$ and the damping ratio is assumed equal to $\zeta=0.0075$ for both modes. The linear frequencies are found to be $\omega_{1}=29.77$ and $\omega_{2}=30.40 \mathrm{rad} / \mathrm{s}$, respectively, associated with the modes $\{1,1\}$ and $\{1,-1\}$. By defining $U=V / \omega_{1} D$, the critical values are $U_{\mathrm{cr} 1}=4.60$ and $U_{\mathrm{cr} 2}=4.69$.

Motion is governed by Eqs. (14). Steady-state solutions $a_{1}^{\prime}=a_{2}^{\prime}=\gamma^{\prime}=0$ are found by numerically solving a three-equation algebraic system. By varying $U$ these solutions describe equilibrium paths in a four-dimensional space $\left\{a_{1}, a_{2}, \gamma, U\right\}$. It is easy to show that the system admits two uncoupled (monomodal) solutions

$a_{1}=\sqrt{\frac{-c_{1}}{3 c_{2}}}, \quad a_{2}=0, \quad \gamma=$ arbitrary,

$a_{1}=0, \quad a_{2}=\sqrt{\frac{-d_{1}}{3 d_{5}}}, \quad \gamma=$ arbitrary,
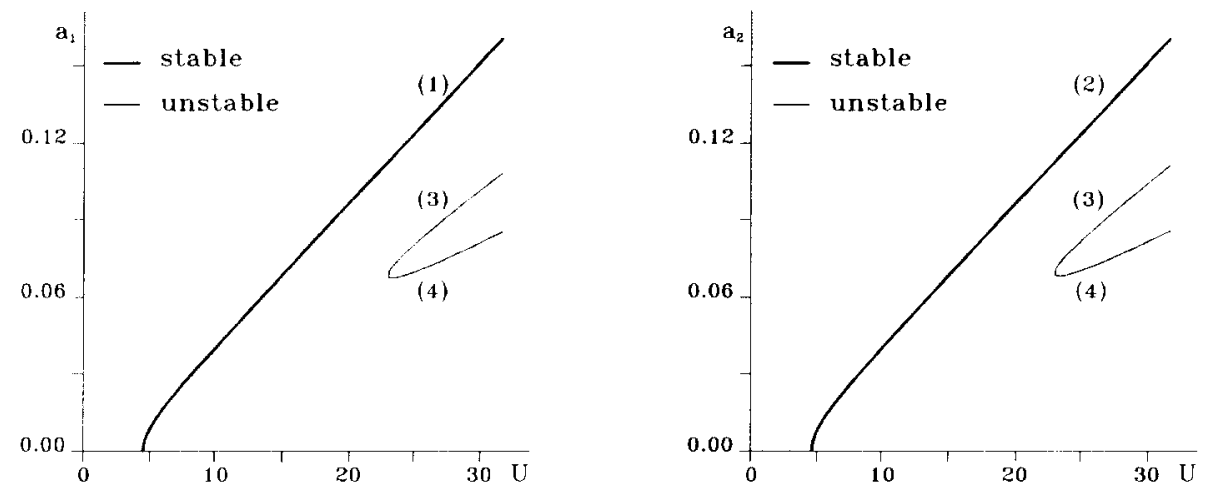

Fig. 2. Steady-state solutions for the symmetrical system. 
that are real for $U>U_{\mathrm{cr} 1}$ and $U>U_{\mathrm{cr} 2}$, respectively. In addition two coupled (bimodal) solutions are found numerically when $U>U_{\mathrm{cr} 1,2}=23.0$. When $U=U_{\mathrm{cr} 1,2}$ the two solutions coalesce. Projections of the equilibrium paths on to the $\left\{a_{1}, U\right\}$ and $\left\{a_{2}, U\right\}$ planes are shown in Fig. 2. Coupled solutions (3) and (4) are found to be saddle points in the state space, i.e. they are unstable; uncoupled solutions (1) and (2) are instead stable.

To analyze the transient response, Eqs. (14) have been numerically integrated starting from different initial conditions. The trajectories obtained have been projected on to the $\left\{a_{1}, a_{2}\right\}$ plane and represented in Fig. 3 for different values of $U$. When $U_{\mathrm{cr} 1}<U<U_{\mathrm{cr} 2}$ (Fig. 3a) there exists only a (stable node)

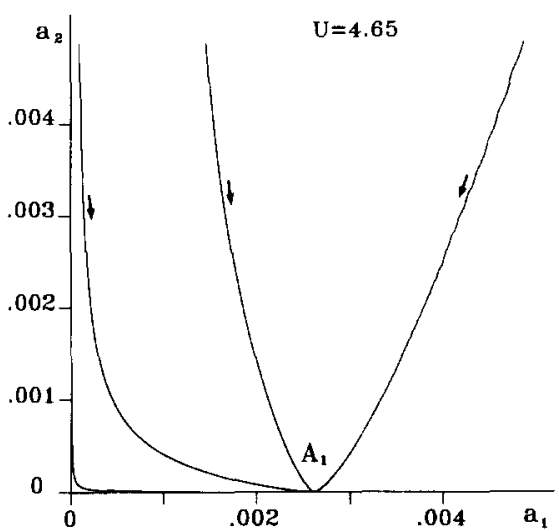

(a)

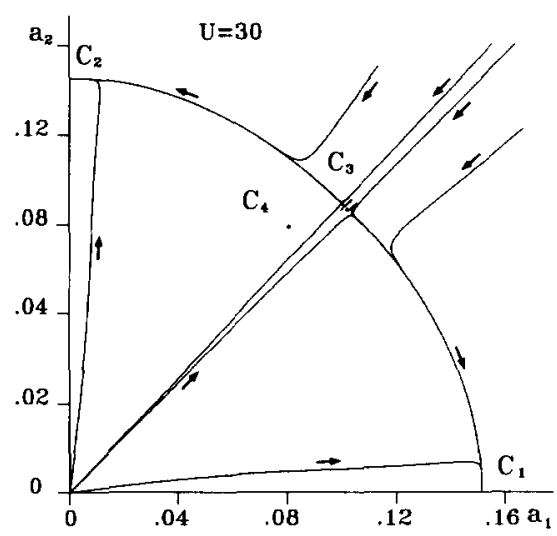

(c)

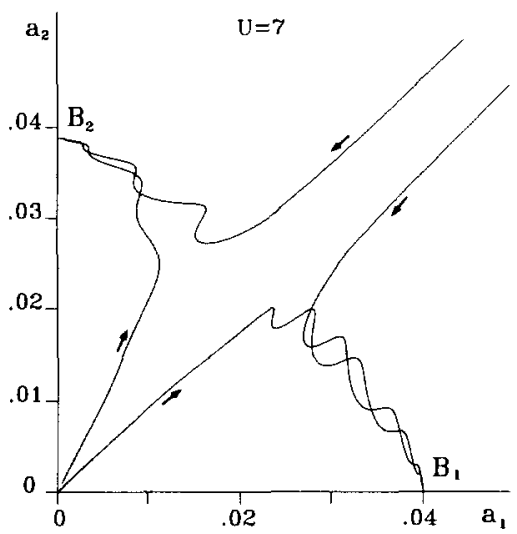

(b)

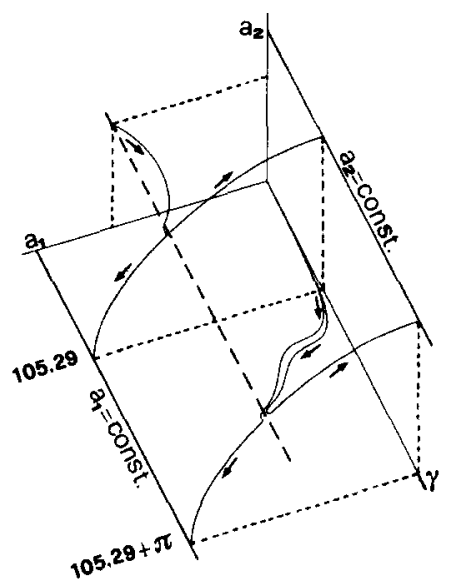

(d)

Fig. 3. Trajectories in the phase plane $\left\{a_{1}, a_{2}\right\}$ and in the phase space $\left\{a_{1}, a_{2}, \gamma\right\}$ : (a) $U_{\mathrm{cr} 1}<U<U_{\mathrm{cr} 2}$, (b) $U_{\mathrm{cr} 2}<U<U_{\mathrm{cr} 1,2}$, (c) $U>U_{\mathrm{cr} 1,2}$, and (d) $U=30$. 
equilibrium point $A_{1}$ on the $a_{1}$-axis attracting all the trajectories. When $U_{\mathrm{cr} 2}<U<U_{\mathrm{cr} 1.2}$ (Fig. $3 \mathrm{~b}$ ) there exist two (stable nodes) equilibrium points $B_{1}$ and $B_{2}$, each on one axis. In this case the trajectories are attracted by either solution, depending on the initial state. Note that the trajectories oscillate around a curve (not shown) joining the two points. Finally, when $U>U_{\mathrm{cr} 1,2}$ (Fig. 3c) there are two (stable nodes) equilibrium points on the two axis, $C_{1}$ and $C_{2}$, and two (unstable saddle) points on the plane, $C_{3}$ and $C_{4}$. All the trajectories are attracted by the monomodal solutions, after having gone along the same nearly elliptical curve.

System evolution is also described in the 3D state-space (Fig. 3d). It is apparent that the representative point moves toward curves which are the intersection of a cylindrical surface and $\gamma=$ constant planes; after having covered the curve, the point reaches an equilibrium point on the $a_{1}$ - or $a_{2}$-axis. In this way one of the two components of the motion decays with a specific phase difference $\gamma$ with respect to the component remaining stationary. The phenomenon has the following energetic interpretation: during the attraction phase to the surface the system stores (or dissipates) energy until a threshold value is reached; after that, while the point describes an elliptical arc, the system transfers energy from one mode to the other. During this phase, and in the subsequent steady-state regime, all the energy put in by the aerodynamic forces is dissipated by the damping.

\subsection{Asymmetrical system}

Let us introduce a small asymmetry in the system by modifying the thickness of the beam $i=2$, in such a way that $I_{2}=1.05 I_{1}, \mu_{2}=1.035 \mu_{1}$, other characteristics remaining unchanged. The frequencies become $\omega_{1}=29.855 \mathrm{rad} / \mathrm{s}$, $\omega_{2}=30.508 \mathrm{rad} / \mathrm{s}$, and the associated modes $\{1,0.72\}$, and $\{-0.74,1\}$; the critical wind velocities are $U_{\mathrm{cr} 1}=4.60$ and $U_{\mathrm{cr} 2}=4.86$.
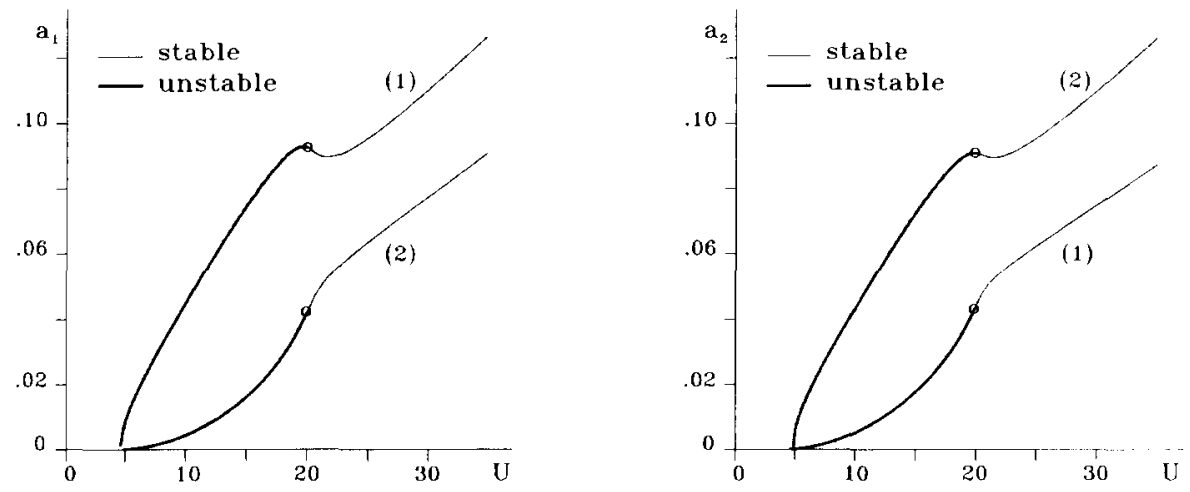

Fig. 4. Steady-state solutions for the asymmetrical systems. 


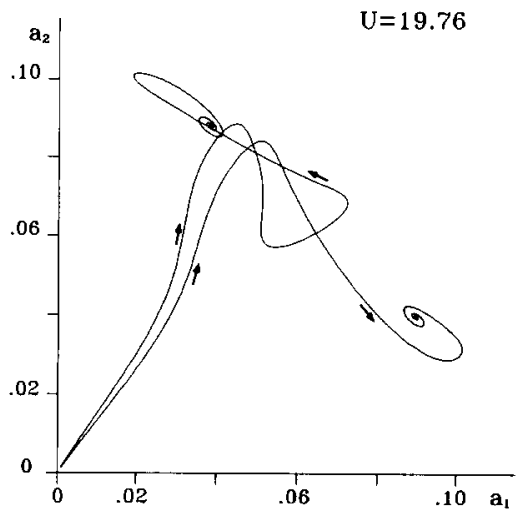

(a)

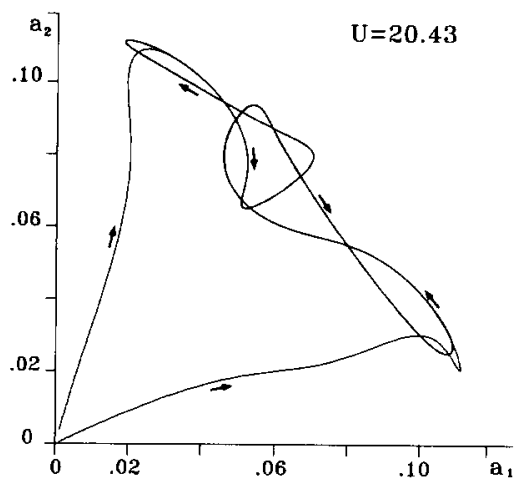

(c)

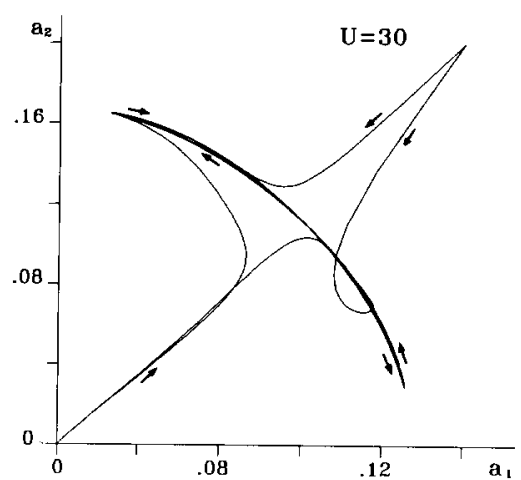

(e)

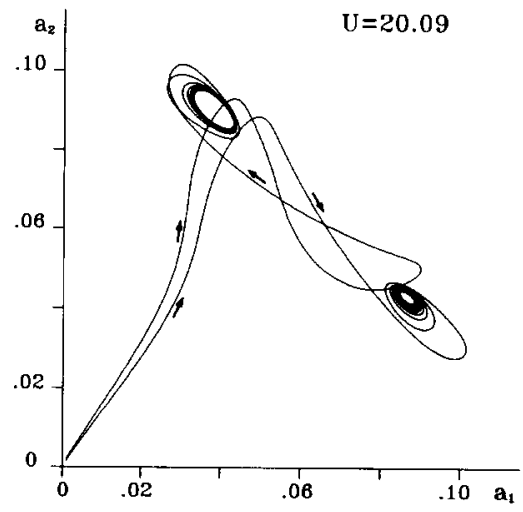

(b)

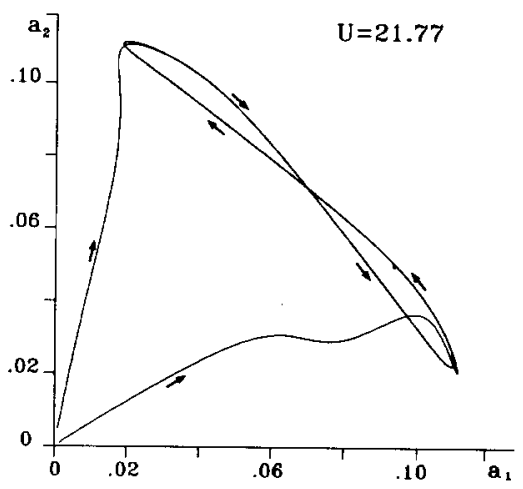

(d)

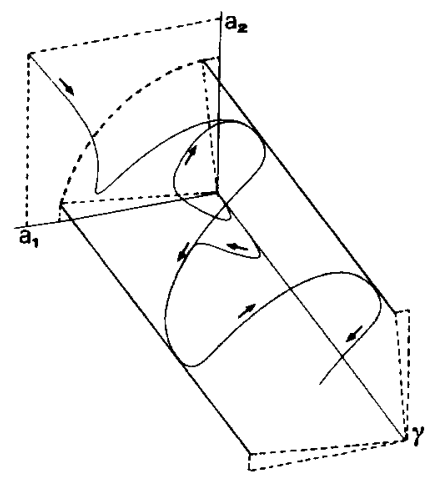

(f)

Fig. 5. Trajectories in the phase plane $\left\{a_{1}, a_{2}\right\}$ and in the phase space $\left\{a_{1}, a_{2}, \gamma\right\}:$ (e) $U=30$. 
Eqs. (13) govern the problem. They do not admit uncoupled solutions. When $U_{\mathrm{cr} 1}<U<U_{\mathrm{cr} 2}$ a unique coupled solution is found, when $U>U_{\mathrm{cr} 2}$ two solutions emerge, as illustrated in Fig. 4. Linear stability analysis has shown that the paths are stable when $U<U_{0} \approx 20.0$ and unstable when $U>U_{0}$. In the critical state $U=U_{0}$ the variational matrix has two imaginary conjugate eigenvalues, the third being real and negative. Thus a Hopf bifurcation occurs and a limit cycle arises.

Some trajectories obtained by numerically integrating Eqs. (13) have been represented in Fig. 5 for different wind velocities. When $U<U_{0}$ (Fig. 5a) the trajectories are attracted by the two (stable foci) bimodal solutions. When $U$ is slightly greater than $U_{0}$ (Fig. 5b), instead of the stable equilibrium points there are two stable limit cycles. By further increasing $U$ (Fig. 5c) the cycles assume an "eight" shape; then a unique cycle exists (Fig. 5d), successively becoming very stretched and lying on an elliptical arc (Fig. 5e). In the 3D state space (Fig. 5f) the trajectory is seen to oscillate on a cylindrical surface. As in the previous case an energetic interpretation holds, but with the difference that the energy is not stored in a unique mode but is continuously exchanged by the two modes, the balance remaining null at any instant.

Curves $a_{1}(t), a_{2}(t)$ relative to the various wind velocities previously considered are plotted in Fig. 6.

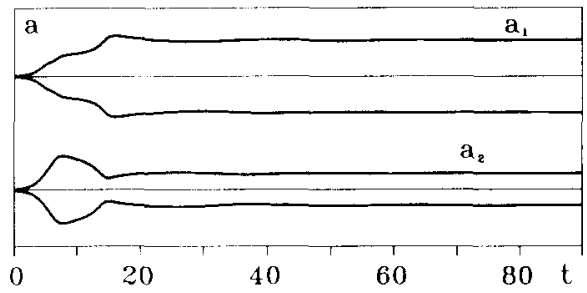

(a)

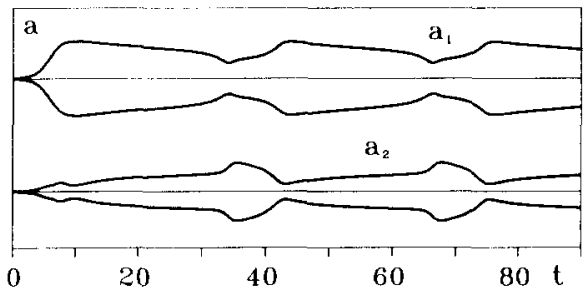

(c)

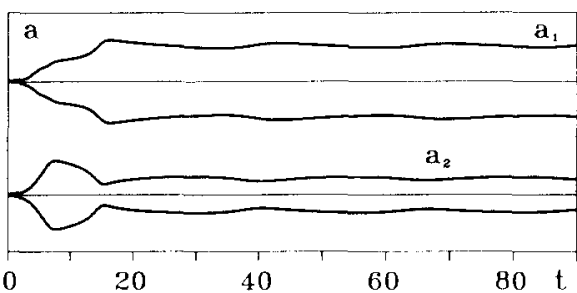

(b)

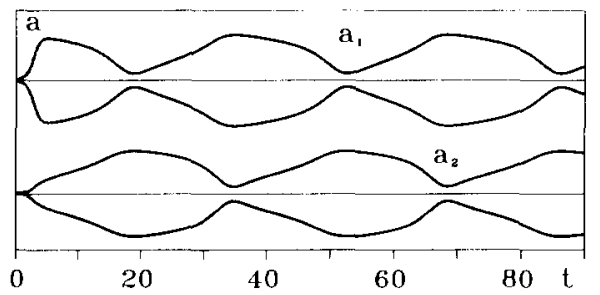

(d)

Fig. 6. Time history responses: (a) $U=19.76$; (b) $U=20.09$ (c) $U=20.43$; and (d) $U=30$. 


\section{Conclusion}

Nonlinear galloping in nearly periodic weakly coupled systems has been analyzed. These systems have narrow band spectra so, when galloping of a mode is excited all remaining modes in the same band are excited too, and combination resonance phenomena occur. The equations governing the amplitude and phase modulation have been formulated for an $n$-elements chain, while numerical results have so far been obtained for a two-element system only. When the two elements are identical there exist monomodal steady-state (unmodulated) solutions, to which the system tends after a transitory phase involving the two components of the motion. On the contrary, when small asymmetries are introduced, only a bimodal solution exists. For critical values of wind velocities a Hopf bifurcation manifests itself and stable limit cycles appear; continuous exchanges of energy between the two modes are then observed.

These preliminary results should be extended to structures in which more than two modes are interacting in the galloping phenomenon.

\section{References}

[1] R.D. Blevins and W.D. Iwan, The galloping response of a two degree of freedom system, J. Appl. Mechanics, 41 (1974) 1113-1118.

[2] D.J. Mead, Wave propagation and natural modes in periodic systems: I. Mono-coupled systems, J. Sound and Vibration, 40 (1) (1975) 1-18.

[3] A.H. Nayfeh, Perturbation Methods (Wiley, New York, 1973).

[4] A.H. Nayfeh and M. Abdel-Rohman, Analysis of galloping responses in cantilever beams, J. Sound and Vibration, 144 (1) (1991) 87-93.

[5] M. Novak, Aeroelastic galloping of prismatic bodies, ASCE, J. Eng. Mechanics Division, 95 (1969) 115-142.

[6] M. Novak, Galloping oscillations of prismatic structures, ASCE, J. Eng. Mechanics Division, 98 (1972) 27-45.

[7] G.V. Parkinson and J.D. Smith, The square prism as an aeroelastic non-linear oscillator, Quarterly, J. Mechanics Appl. Mathematics, 17 (1964) 225-239.

[8] P. Pheinsusom, Y. Fujino and M. Ito, Galloping of tower-like structure with two closely spaced natural frequencies, J. Wind Eng. Ind. Aerodyn., 32 (1989) 189-198.

\section{Appendix}

Coefficients $c_{i}$ and $c_{i j k l}$ appearing in Eqs. (3) are

$$
c_{i}=-2 \zeta_{i} \omega_{i}+\frac{1}{2} \frac{\rho V \sum_{h=1}^{n} D_{h} \mathscr{A}_{1 h} u_{i h}^{2} l_{h}^{2} \int_{0}^{1} \psi^{2} \mathrm{~d} x_{h}}{\sum_{h=1}^{n} u_{i h}^{2} l_{h}^{2} \int_{0}^{1} \mu_{h} \psi^{2} \mathrm{~d} x_{h}}
$$


$c_{i j k l}=\frac{1}{2} \frac{\rho V^{-1} \sum_{h=1}^{n} D_{h} \mathscr{A}_{3 h} u_{i h} u_{j h} u_{k h} u_{l h} l_{h}^{4} \int_{0}^{1} \psi^{4} \mathrm{~d} x}{\sum_{h=1}^{n} u_{i h}^{2} l_{h}^{2} \int_{0}^{1} \mu_{h} \psi^{2} \mathrm{~d} x}$,

where $\zeta_{i}$ is the damping ratio of the $i$-th mode, $\mu_{h}$ is the mass density of the $h$-th beam and $u_{i h}$ the $h$-th component of the $i$-th mode, other symbols being defined in the text. Coefficients $c_{i}$ and $d_{i}$ appearing in Eqs. (13) and (14) are

$$
\begin{aligned}
c_{1} & =\frac{\rho V D \mathscr{A}_{1}}{\mu}-\zeta_{1} \omega_{1} & d_{1} & =\frac{\rho V D \mathscr{d}_{1}}{\mu}-\zeta_{2} \omega_{2} \\
c_{2} & =\frac{1}{8 \omega_{1}} c_{1111} \omega_{1}^{3} & d_{2} & =\frac{1}{8 \omega_{2}} c_{2111} \omega_{1}^{3} \\
c_{3} & =\frac{3}{8 \omega_{1}} c_{1211} \omega_{1}^{2} \omega_{2} & d_{3} & =\frac{3}{8 \omega_{2}} c_{2211} \omega_{1}^{2} \omega_{2} \\
c_{4} & =\frac{3}{8 \omega_{1}} c_{1221} \omega_{1} \omega_{2}^{2} & d_{4} & =\frac{3}{8 \omega_{2}} c_{2221} \omega_{1} \omega_{2}^{2} \\
c_{5} & =\frac{1}{8 \omega_{1}} c_{1222} \omega_{2}^{3} & d_{5} & =\frac{1}{8 \omega_{2}} c_{222} \omega_{2}^{3}
\end{aligned}
$$

